\title{
The Passion, Beauty, and Joy of Teaching and Learning Cybersecurity
}

\author{
Richard Weiss \\ (moderator) \\ Evergreen State College \\ 2700 Evergreen Pkwy NW \\ Olympia, WA \\ 1-360-867-6871 \\ weissr@evergreen.edu
}

\author{
Xenia Mountrouidou \\ College of Charleston \\ 66 George St. \\ Charleston, SC \\ 1-843-953-2754 \\ mountrouidoux@cofc.edu
}

\author{
Jens Mache \\ Lewis \& Clark College \\ 0615 SW Palatine Hill Rd \\ Portland, OR \\ 1-503-768-7564 \\ jmache@Iclark.edu
}

\section{CCS Concepts}

- Security and protection - Computer Science Education

\section{Keywords}

Cybersecurity; Assessment; Cloud computing; CTF

\section{SUMMARY}

In the ACM/ IEEE Model Curriculum of 2013, cybersecurity became a new core knowledge area. According to a Cisco report [1] there will be one million jobs in the cybersecurity field that will go unfilled. Currently, cybersecurity education is struggling to keep up not only with producing more experts in the field, but also with the fast pace of technology evolution, so it is difficult for faculty to teach it. Nevertheless, it is exciting and relevant, and there are many experiential ways to teach it rather than reading a standard textbook. In addition to addressing the need for more students, we also want a more diverse workforce.

This panel presents several approaches to revealing the excitement and relevance of the field to more students. Our suggestions address three aspects:

1) Passion- making the study rewarding for everybody through puzzles and games,

2) Beauty - making the study inviting and relevant through interdisciplinary topics, and

3) Joy - making teaching hands-on exercises easy to access \& assess (through VMs in the cloud).

We will be presenting three diverse solutions to teaching Cybersecurity with current, emerging technologies and making it appealing to undergraduate students.

The first approach is using Capture the Flag Competitions (CTFs) and industry aligned curriculum to bring the passion in learning about cybersecurity. Now in its fifth year, the National Cyber League (NCL) [5] has a powerful and proven model - provide an ongoing virtual training ground for faculty and students to develop and validate cybersecurity skills using content aligned with individual and team games - which is scalable across many industry certifications, curricula, job roles, and verticals.

Permission to make digital or hard copies of part or all of this work for personal or classroom use is granted without fee provided that copies are not made or distributed for profit or commercial advantage and that copies bear this notice and the full citation on the first page. Copyrights for third-party components of this work must be honored. For all other uses, contact the Owner/Author.

Copyright is held by the owner/author(s)

SIGCSE '17, March 08-11, 2017, Seattle, WA, USA.

ACM 978-1-4503-4698-6/17/03.

http://dx.doi.org/10.1145/3017680.3017692
The second approach is CyberPaths, creating a new paradigm for teaching Cybersecurity in the liberal arts, with general education modules related to law, policy, international conflict, management, and human factors. Furthermore, realistic experimentation on GENI [2] attracts a diverse student population from liberal arts institutions, eliminating the need for creating and maintaining local infrastructure. The project designs different paths for students to double major or minor in CS or complete a simple concentration in Cybersecurity, in accordance with the multidisciplinary education in liberal arts colleges.

The third approach is EDURange [4], which addresses ease of use for how we teach Cybersecurity experientially. Furthermore, EDURange offers automated tools that keep track of the thinking process when a student is solving a cybersecurity problem. This offers better evaluation methods and constructive feedback to the students.

\section{CASEY O'BRIEN}

The NCL [5] was founded in May 2011 to provide an ongoing virtual training ground for collegiate students to develop, practice, and validate their cybersecurity skills using next-generation highfidelity simulation environments.

The NCL has been gamifying learning to make it exciting and nontraditional through exploration and constructive competition. NCL provides students with feedback on how they did in the challenges.

One of the distinguishing factors of the NCL is the integration of learning objectives in all its activities. One of the main ways this is accomplished is by aligning customized content available in NCL Gymnasiums (virtualized learning environments) with simulations and games. This allows players to use the Gym environment to develop knowledge and skills and then demonstrate these newly acquired skills in competitive individual and team play. It also allows the NCL to measure a player's game performance and produce individualized reports (NCL Scouting Report) with strengths and weakness for various learning objectives and industry-recognized competencies.

The 2016 Fall Season was by far the largest event the NCL has run, and is the largest paid game of its type in the world. The 2016 Fall Season included:

- Record numbers of participants

- Continued corporate sponsor (Facebook)

- Provided students with diverse skill levels, fun, and engaging learning and playing environments available anywhere 
- Measured how players perform in the games and produced individualized Scouting Reports (performance-based assessments)

- Created school pride for players and coaches

\section{XENIA MOUNTROUIDOU}

The project CyberPaths offers a new paradigm for teaching security in liberal arts colleges. First, we integrate Cybersecurity teaching non-intrusively throughout the general education curriculum, to demonstrate that it is a truly an interdisciplinary field and to attract a diverse population of non-CS majors. Second, we use the Global Environment for Network Innovations (GENI), to teach Cybersecurity experientially with low overhead in primarily undergraduate institutions with limited resources.

We have devised modules that can be integrated into policy, law, finance, and humanities courses. These modules demonstrate how different disciplines connect to Cybersecurity. Specifically, the policy module involves laws, international conflict, and cyberwar concepts in role play and research exercises. The finance module demonstrates how to invest in Cybersecurity with the GordonLoeb model [3]. The humanities module discusses privacy and human factors in security.

After students complete these modules, if they are more interested in Cybersecurity they can take a Security 101 class without prerequisites. This class is specifically designed to demonstrate the interdisciplinary nature of the field and will include active learning labs that we have devised on GENI. Additional paths are open to students that want more specialization, through a concentration in Cybersecurity with a minor or a double major in $\mathrm{CS}$, in accordance with the Liberal Arts education that promotes well rounded citizens.

We will present the material that we have created on the GENI infrastructure, general education modules, and some preliminary results experimenting in a quantitative reasoning general education class. Students reported that after completion of the Cybersecurity modules they were more interested in exploring CS and taking more courses in the field.

\section{JENS MACHE and RICHARD WEISS}

EDURange is making teaching hands-on exercises easy to access through VMs in the cloud (using Amazon's EC2) [6]. No reservations are required. No software needs to be installed - one only needs a browser and an ssh-client.

Many games have automated scoring, but scoring is not the same as assessment. Assessment should provide feedback to students, so that they can correct their mistakes and learn new material efficiently. Assessment for hands-on exercises is both important and difficult. We want to reach large numbers of students, and we want to engage them. The first step is to provide interesting challenges, which EDURange does[6], but then we need to give students feedback on how they are doing. This is an important role for faculty and is not something we automate. Instead, EDURange provides tools to visualize what students are doing as they work on the exercises [7]. This allows instructors to more easily see when students are stuck or heading in the wrong direction. Since cybersecurity exercises are often easy to understand but hard to solve, guidance is especially important so that students don't become frustrated.

Gamification can turn hard problems into challenging exercises that are interesting to students. As the field of cybersecurity changes, we need to continue to create new exercises that are exciting and can be used in the classroom. EDURange [4] provides a framework for creating new exercises and tools to help with their assessment.

\section{ACKNOWLEDGEMENTS}

This work was supported in part by the National Science Foundation under grants 1600060, 1516730, 1516100, and by Amazon. We thank Dan Garcia for his wonderful series of panels Rediscovering the Passion, Beauty, Joy and Awe, which inspired our title [8].

\section{REFERENCES}

[1] Cisco. Cybersecurity Talent Gap. http:/www.cisco.com/c/dam/en/us/products/collateral/securit $\mathrm{y} /$ cybersecurity-talent.pdf

[2] Global Environment for Network Innovations (GENI). http://www.geni.net/

[3] Gordon, L. A., and Loeb, M. P. The economics of information security investment, Journal of ACM Transactions on Information and System Security (TISSEC), Volume 5 Issue 4, November 2002, Pages 438-457

[4] EDURange, http://edurange.org

[5] NCL, http://www.nationalcyberleague.org/

[6] Weiss, R., Boesen, S., Sullivan, J. F., Locasto, M. E., Mache, J., and Nilsen, E. Teaching cybersecurity analysis skills in the cloud. In Proceedings of the 46th ACM Technical Symposium on Computer Science Education (2015), ACM, pp. 332-337. DOI= http://dx.doi.org/10.1145/2676723.2677290

[7] Weiss, R., Locasto, M. E., and Mache, J. A reflective approach to assessing student performance in cybersecurity exercises. In Proceedings of the 47th ACM Technical Symposium on Computing Science Education (2016), ACM, pp. 597-602. DOI= http://dx.doi.org/10.1145/2839509.2844646

[8] Garcia, D. D., Cutler, R., Dodds, Z., Roberts, E. and Young, A. Rediscovering the passion, beauty, joy, and awe: making computing fun again, continued. SIGCSE Bull. 41, 1 (March 2009), 65-66.

DOI=http://dx.doi.org/10.1145/1539024.1508889 